\title{
Formación universitaria, migraciones e interculturalidad en España: una revisión de la oferta educativa de los estudios de Grado de Educación Infantil, Educación Primaria, Pedagogía y Educación Social
}

University Education, Migrations And Interculturality In Spain: A Review Of Educational Provision For The Degree In Early Childhood Education, Elementary Education, Pedagogy And Social Education

María Rubio Gómez, Raquel Martinez Chicón y Antonia Olmos Alcaraz ${ }^{1}$

\section{Resumen}

Este trabajo constituye una revisión de las diversas respuestas que las universidades españolas están ofreciendo en sus planes de estudios y contenidos curriculares a la realidad multicultural. Concretamente nos centramos en el diseño de la formación pensada para futuras/os profesionales del ámbito educativo formal y no formal. El objetivo del trabajo ha sido obtener un primer diagnóstico sobre cómo está siendo la formación universitaria reglada sobre «migraciones» e «interculturalidad» para maestras/os, pedagogas/os y educadoras/es sociales en España. Para ello, a lo largo del texto tratamos de responder a las siguientes preguntas: ¿Se está ofreciendo una formación adecuada para intervenir en los nuevos contextos de creciente diversidad cultural? ¿En qué universidades españolas?, ¿qué características presenta dicha formación? Y en esta primera aproximación, los resultados aportan una visión bastante clarificadora de la realidad de la «formación intercultural» en la Universidad. Existe una oferta amplia de asignaturas en los grados de Educación Infantil, Educación Primaria, Pedagogía y Educación Social que contemplan en sus programas la formación en migraciones e interculturalidad y que dan cobertura al estudio y análisis de la diversidad cultural desde distintos enfoques disciplinares y en muchas ocasiones innovadores, pero, en la mayoría de los casos, se trata de asignaturas optativas que se ven relegadas a la libre elección del alumnado.

\section{Palabras clave}

Universidades, sociedad, formación inicial, educación intercultural, migraciones.

\section{Abstract}

This paper is a review on how Spanish universities are tackling multicultural reality in their curricula in a variety of ways. In particular, we focus on the training program for future professionals of the formal and non-formal educational field. The aim of this work, is to obtain a first assessment on the formal university programs about «migrations» and «interculturality» for teachers, pedagogues and social educators in Spain. To this effect, in this text, we have tried to get responses to the following questions: Are the universities offering the right training to intervene within the new increased cultural diversity contexts? Which universities are these ones? What are the main characteristics of these trainings? In this first approach, the results provide us with a very enlightening insight into the reality of «intercultural training» at university. There is a wide range of subjects in the Early Childhood Education degree, Bachelor of Education degree, Pedagogy degree and Social Education degree which provide training on migrations and multiculturalism. These subjects are well covering the analysis of cultural diversity from different and innovative interdisciplinary approaches, but, in most cases are electives which means are left to the student's choice.

Keywords

Universities, society, initial training, intercultural education, migration.

\section{Cómo citar/Citation}

Rubio Gómez, María; Martínez Chicón, Raquel; Olmos Alcaraz, Antonia (2019). Formación universitaria, migraciones e interculturalidad en España: una revisión de la oferta educativa de los estudios de Grado de Educación Infantil, Educación Primaria, Pedagogía y Educación Social. Revista de Sociología de la Educación-RASE, 12 (2), 337-350. http://dx.doi.org/10.7203/RASE.12.2.14655.

\footnotetext{
1 María Rubio Gómez, Universidad de Granada, mariarubio@ugres; Raquel Martinez Chicón, Universidad de Granada, raquelchicon@ugr.es; Antonia Olmos
} Alcaraz, Universidad de Granada, antonia@ugr.es. 


\section{Introducción}

España es un país de recepción de flujos migratorios desde principios de la década de los años noventa del pasado siglo. A partir de entonces, y desde presente milenio especialmente, las distintas administraciones públicas han desplegado políticas de gestión de la diversidad encaminadas a intervenir en distintos ámbitos de la sociedad. El espacio educativo ha sido uno de estos ámbitos de intervención, y donde mejor podemos observar cómo, poco a poco, se están introduciendo enfoques de carácter multi e intercultural. También es el espacio desde donde surge y en el que se analiza todo el debate académico sobre el uso de los conceptos de interculturalismo y multiculturalismo, los abusos del concepto de «cultura» y sus repercusiones en la gestión de la diversidad o en la gestión de las diferencias -y desigualdades- culturales (García Castaño et al., 2012; Olmos y Martínez, 2015).

El trabajo que se presenta constituye una primera aproximación a la temática en el espacio universitario. Una revisión sobre cómo las universidades españolas están respondiendo, con sus planes de estudios y contenidos curriculares, a esta realidad multicultural en el diseño de la formación ofrecida a futuras/os profesionales del ámbito educativo formal y no formal, que trabajarán en todos los casos en contextos multiculturales. Para ello tratamos de responder a las preguntas siguientes: ¿se está ofreciendo formación inicial a futuras/os profesionales del magisterio, la pedagogía y la educación social para intervenir en espacios de creciente diversidad cultural? y ¿qué características presenta dicha formación? Pero antes de continuar es preciso realizar un inciso sobre cómo surgió y cómo se está desarrollando el enfoque intercultural en España, dado que hemos der ser conscientes de que los contextos imprimen particularidades concretas a los paradigmas, en este caso, al paradigma intercultural.

Es a partir de 1990 cuando en este país comienza a hablarse de interculturalismo (García Castaño y Pulido, 2008) y, dentro de este enfoque, de Educación Intercultural en el contexto educativo, llegando al extremo de lo que Dietz (2012) denomina la «pedagogización de la interculturalidad» que acaba dejando a un lado un verdadero análisis y debate sobre el interculturalismo en España. Esto sucede en paralelo a la llegada de población inmigrante extranjera al país, lo que ha llevado a identificar en exceso el fenómeno migratorio con el enfoque intercultural. En otras palabras, si bien -y nos centramos ya en la Educación Intercultural- sabemos que la gestión de la diversidad cultural no hace referencia sólo y exclusivamente a la intervención en contextos con presencia de poblaciones identificadas como inmigrantes, la realidad es que en España es lo que precisamente se hace. Ello redunda en una noción de diversidad cultural limitada, que en el plano educativo se ha materializado en la puesta en práctica de una Educación Intercultural enfocada a la atención/actuación con alumnado que cumple unas determinadas características (Franzé Mudanó, 2003; Olmos Alcaraz 2010; García Castaño et al., 2011; Carrasco Pons, 2011). Debido a ello, la aproximación que hemos realizado aborda la formación sobre «interculturalidad»y «migraciones»en los estudios universitarios de Grado de Educación Infantil, Educación Primaria, Pedagogía y Educación Social en las universidades públicas españolas. Al margen quedan las críticas que podamos hacer, y que de hecho hacemos, sobre el reduccionismo que ello implica al olvidar «otras diversidades» que conforman la multiculturalidad contemporánea en el país.

El objetivo del trabajo ha sido obtener un diagnóstico sobre cómo está siendo la formación universitaria reglada sobre «interculturalidad» y/o «migraciones», orientada a la formación inicial de maestras / os, pedagogas/os y educadoras/es sociales en el país, en tanto que profesionales que intervendrán en un futuro próximo sobre la presente realidad multicultural en el ámbito educativo. Todo ello, entendido como una forma de innovación docente a nivel universitario porque, -e insistimos-a pesar de las críticas 
de reduccionismo, es cierto que, hasta hace bien poco, el paradigma imperante en el sistema educativo español no dejaba apenas opción al tratamiento de las diversidades.

La gran mayoría de las investigaciones sobre prácticas «interculturales» y muchos de los programas de atención a la diversidad en las escuelas surgieron a raíz del reconocimiento de la diversidad cultural -en términos de diferencia y de atención diferenciada, compensatoria y, en muchas ocasiones, desigual- por la presencia de población gitana y posteriormente de población inmigrante extranjera en las aulas (García, Granados y Pulido, 1999). En la mayoría de los casos no sólo las prácticas fueron previas, sino que fueron precisamente estas, y sus análisis, las que pusieron de manifiesto la necesidad de formar al profesorado en «interculturalidad». Así, ya encontramos a finales de los años noventa del pasado siglo reivindicaciones desde los espacios científicos y académicos (García, Granados y García-Cano, 1999) sobre la necesidad de que la dimensión intercultural y la comprensión de las diferentes (y diversas) comunidades de la sociedad fuesen objeto de estudio y formaran parte de la formación inicial y permanente del profesorado. Por otro lado, también se insistía en que esta formación no se centrara en sensibilidades, motivaciones o «voluntarismos personales» (Jordán, 1992), que suplieran la falta de competencias técnicas.

Aunque los trabajos e investigaciones sobre la educación intercultural indiquen la necesidad de ampliar su alcance más allá del profesorado, casi la totalidad de ellos lo consideran pieza clave y la gran mayoría coincide en sus conclusiones en que la formación del profesorado debe ser fundamental y estar integrada en los planes de estudio como parte de las disciplinas obligatorias. Sin embargo, y de manera contraria, dicha formación inicial se plantea generalmente como optativa y puntual, no suele ser una formación de carácter permanente y continua y tiende a centrarse en planteamientos y enfoques compensatorios y de educación especial (Rodríguez Izquierdo, 2009).

Sigue existiendo en la actualidad una enorme diversidad de propuestas concretas respecto a los aspectos a trabajar y a las competencias que adquirir por parte de los profesionales del sector educativo y que deberían estar presentes en cualquier programa de formación en interculturalidad. Sin entrar a detallar los diferentes modelos propuestos desde los inicios de la educación intercultural y de la formación en interculturalidad, en lo que coinciden la casi totalidad de ellos es en la necesidad de desarrollar determinadas «competencias interculturales», entendiendo por estas el conjunto de conocimientos, actitudes y habilidades necesarias para el desempeño exitoso de su labor en contextos de diversidad cultural; y que contienen tres dimensiones fundamentales: la cognitiva -conocimiento: cultural, contextual y estructural del «nosotros» primero y del «ellos» después-, la afectiva -empatía- y la comportamental -habilidades comunicativas y de relación- (Rodrigo, 1997, 1999; Vázquez, 2009; Martínez 2011; Martínez y Olmos, 2014). Esta adquisición de competencias que debe ser objetivo de cualquier formación en interculturalidad y migraciones, sea o no reglada, tal y como recoge Leiva $(2012,2014)$ y plantean Peñalva y Soriano (2010), no puede centrarse exclusivamente en el profesorado, sino que debe dirigirse a toda la comunidad educativa. Esto implica no sólo un enfoque formativo democrático e inclusivo (Essomba, 2006), sino un enfoque holístico. Y añadimos, en contextos profesionales, sea o no reglada, debería ser una formación continua y permanente, como la ya existente por iniciativa de algunas administraciones públicas como es el caso de la andaluza con los programas formativos FORINTER: Formación en Interculturalidad y FORINTER2: Formación en Interculturalidad y Migraciones; centrados en la adquisición de competencias interculturales para empleados/as públicos, entre los que destaca los del ámbito educativo. (Consejería de Gobernación, 2006 y Consejería de Empleo, 2009). Pero en los contextos formativos de educación superior debería ser además una formación reglada de carácter obligatorio que permitiera dejar de repro- 
ducir enfoques compensatorios al diseñarse, en no pocas ocasiones, desde visiones de la diversidad como diferencias a compensar (García, Rubio y Echeverría, 2018).

\section{Metodología}

El proceso metodológico seguido para la realización de este trabajo ha consistido en la revisión de todos los planes de estudio de las universidades de titularidad pública ${ }^{2}$ de las distintas Comunidades Autónomas españolas (48 en total). Para ello se han consultado las webs de todas las universidades, y la información que al respecto aparecía en las mismas. Es por ello, y por constituir este trabajo una primera aproximación general, que la información que aquí se muestra se limita a ofrecer los datos públicos que figuran en las páginas web de las universidades. En este proceso se han identificado el número de universidades que imparten estudios de grado en el campo de las Ciencias de la Educación (en concreto los estudios de Grado de Educación Infantil, Grado de Educación Primaria, Grado de Pedagogía y Grado de Educación Social), 41 en total; y cuántas/cuáles de ellas introducen en sus planes de estudio materias relacionadas con la temática abordada («interculturalidad»y/o «migraciones»), 35 en total. A continuación, se han analizado estas asignaturas teniendo en cuenta el nombre, el carácter obligatorio u optativo, el número de créditos, los cursos en los que se imparten y los departamentos a los que pertenecen.

Los criterios seguidos para la inclusión o no de las asignaturas en la muestra analizada han sido la presencia en el título de la materia de alguno de estos términos: migraciones, interculturalidad, multiculturalidad y diversidad cultural. Se han omitido por lo tanto muchas asignaturas que posiblemente -aunque no contemplen en el título dichas temáticas- en sus contenidos pueden estar abordando el tema. Es el caso de asignaturas como Sociología de la Educación y Antropología de la Educación, entre otras. También se ha evitado incluir en la muestra las materias que contenían en su título referencias a la «diversidad» en sentido amplio (sin ir seguida de la palabra cultural: «diversidad cultural»). El motivo de esta exclusión es que dichas materias (que suelen tener títulos como «Atención a la Diversidad»), en gran medida están enfocadas a la atención de diversidades que tienen que ver con la diversidad funcional, y así se ha comprobado al revisar los contenidos de las mismas. Por ello, pese a que es posible que en algunas de ellas se aborden todo tipos de diversidades, no han sido incluidas en el estudio.

\section{Presentación y análisis de resultados: Migraciones e interculturalidad en grados sociales}

En el análisis realizado se ha trabajado sobre los planes de estudios de 41 universidades públicas, que ofrecen desde 1 a 4 de los grados seleccionados para realizar la observación. Los grados que en más universidades se ofertan son, en primer lugar, los de Educación Infantil y Primaria (se ofertan, ambos, en 39 de las 41 universidades de la muestra); en segundo lugar, Educación Social (en 30 de las 41 universidades); y por último el Grado de Pedagogía (en 19 de las universidades observadas). Como se muestra en el Cuadro 1 , $\tan$ solo 6 (el 14,6\%) de las universidades no presentan asignaturas sobre las materias observadas aun ofertando algunos de los estudios de grado considerados. El resto (el 85,4\%, 35 universidades) sí

\footnotetext{
2 Hemos escogido solo universidades públicas por cuestiones relacionadas con el alcance (en términos de número de alumnado) y con la orientación en cuanto a los objetivos, visión, misión etc. En este sentido, y en relación a la primera cuestión mencionada (el alcance) un trabajo reciente de De la Torre (2018) nos indica que aun suponiendo el 40\% del sistema universitario español, las universidades privadas en el curso 2016/17 solo atendieron al 14\% de los estudiantes universitarios; por lo que su alcance en términos cuantitativos es bastante reducido. En segundo lugar, los diversos modelos de gestión que universidades públicas y privadas presentan han sido también motivo para centrarnos solo en universidades pública cuya orientación está más basada en "criterios más relacionados con la provisión de un servicio público a la sociedad" (De la Torre, 2018), por lo que desde esta lógica es coherente reivindicar la necesaria capacitación intercultural de futuros profesionales sociales que la educación superior pública debe garantizar.
} 
recogían en alguno -o varios- de los grados seleccionados asignaturas sobre «interculturalidad» y/o «migraciones». Este es un primer dato a resaltar: un porcentaje elevado de las universidades públicas españolas que ofrecen los grados de Educación Infantil, Primaria, Pedagogía y/o Educación Social contemplan en la actualidad en sus planes educativos alguna/s materia/s sobre «interculturalidad» y/o «migraciones».

Cuadro 1. Universidades públicas españolas con oferta de asignaturas sobre «interculturalidad» y/o «migraciones» explícitas en su título en grados del área de Ciencias de la Educación (Curso 2018-19). 1 = Se oferta, 0 = no se oferta; s.d. = no hay datos

\begin{tabular}{|c|c|c|c|c|}
\hline UNIVERSIDAD & GRADO INFANTIL & GRADO PRIMARIA & GRADO PEDAGOGÍA & $\begin{array}{l}\text { GRADO EDUCACIÓN } \\
\text { SOCIAL }\end{array}$ \\
\hline U. Almería & 1 & 0 & s.d. & 1 \\
\hline U. Jaén & 1 & 1 & s.d. & 0 \\
\hline U. Córdoba & 1 & 1 & s.d. & 1 \\
\hline U. Granada & 1 & 0 & 1 & 1 \\
\hline U. Huelva & 1 & 0 & s.d. & 1 \\
\hline U. Málaga & 0 & 1 & 0 & 1 \\
\hline U. Pablo de Olavide & s.d. & s.d. & s.d. & 1 \\
\hline U. Sevilla & 0 & 1 & 0 & s.d. \\
\hline U. Cádiz & 0 & 0 & s.d. & s.d. \\
\hline U. Zaragoza & 1 & 1 & s.d. & s.d. \\
\hline U. Las Palmas de Gran Canaria & 0 & 1 & s.d. & 1 \\
\hline U. de La Laguna & 0 & 0 & 0 & s.d. \\
\hline U. Cantabria & 0 & 1 & s.d. & s.d. \\
\hline U. Castilla-La Mancha & 0 & 1 & s.d. & 1 \\
\hline U. Burgos & 1 & 1 & 1 & 1 \\
\hline U. León & 1 & 0 & s.d. & 1 \\
\hline U. Salamanca & 1 & 0 & 1 & 1 \\
\hline U. Valladolid & 1 & 1 & s.d. & 1 \\
\hline U. Autónoma de Barcelona & 0 & 0 & 0 & 1 \\
\hline U. Barcelona & 1 & 1 & 0 & 0 \\
\hline U. Girona & 1 & 1 & 1 & 1 \\
\hline U. Leida & 1 & 0 & s.d. & 1 \\
\hline U. Rovira I Virgili & 1 & 1 & 1 & 1 \\
\hline U. Alcalá & 1 & 1 & s.d. & 1 \\
\hline U. Complutense de Madrid & 1 & 0 & 0 & 1 \\
\hline U. Autónoma de Madrid & 0 & 0 & s.d. & 0 \\
\hline U. Rey Juan Carlos & 0 & 0 & s.d. & s.d. \\
\hline UNED & s.d. & s.d. & 1 & 1 \\
\hline U. Navarra & 1 & 1 & 1 & s.d. \\
\hline U. La Rioja & 0 & 1 & s.d. & s.d. \\
\hline U. Valencia & 0 & 0 & 1 & 0 \\
\hline U. Alicante & 0 & 0 & s.d. & s.d. \\
\hline U. Jaume I de Castellón & 0 & 0 & s.d. & s.d. \\
\hline U. Extremadura & 0 & 0 & s.d. & 1 \\
\hline U. A Coruña & 1 & 1 & s.d. & 1 \\
\hline U. Santiago de Compostela & 0 & 0 & 1 & 1 \\
\hline U. Vigo & 0 & 0 & s.d. & 1 \\
\hline U. Islas Baleares & 0 & 1 & 1 & 1 \\
\hline
\end{tabular}




\begin{tabular}{|c|c|c|c|c|}
\hline UNIVERSIDAD & GRADO INFANTIL & GRADO PRIMARIA & GRADO PEDAGOGÍA & $\begin{array}{c}\text { GRADO EDUCACIÓN } \\
\text { SOCIAL }\end{array}$ \\
\hline U. País Vasco & 1 & 1 & 0 & 1 \\
\hline U. Oviedo & 0 & 0 & 1 & s.d. \\
\hline U. Murcia & 0 & 1 & 1 & 0 \\
\hline
\end{tabular}

Fuente: elaboración propia.

Si establecemos una comparación entre el número de universidades que ofrecen cada uno de los grados considerados y los datos sobre el número de asignaturas relacionadas con las materias estudiadas, vemos cómo el Grado de Educación Social es el que, en mayor medida, está dando cobertura a esta temática (en 24 de las 30 universidades que ofrecen el grado hay alguna asignatura sobre estas cuestiones, pudiendo contar un total de 39 asignaturas). Como se muestra en la Tabla I, de dichas asignaturas solo el 30\% (12 del total) son obligatorias y entre todas representan tan solo el 4,9\% del total de los créditos ofertados por dichas universidades para el grado mencionado, oscilando entre el 1,2\% de la Universidad de Girona (con solo una asignatura optativa de 3 créditos en esta línea) y la Universidad Rovira i Virgili en la que se ofertan 5 asignaturas optativas de 6 créditos, es decir, 12,5\% del total de créditos del grado.

\section{Tabla I. Asignaturas sobre «interculturalidad» y/o «migraciones» ofertadas para los estudios de Grado de Educación Social en las universidades públicas españolas (Curso 2018-19)}

\begin{tabular}{|c|c|c|c|}
\hline UNIVERSIDAD & ASIGNATURAS & CARÁCTER & CRÉDITOS \\
\hline U. Almería & 1. Deporte, multiculturalidad e integración social & $O P$ & 6 \\
\hline \multirow{2}{*}{ U. Córdoba } & 2. Migraciones, interculturalidad y gestión de la diferencia & OP & 6 \\
\hline & 3. Idiomas para la cohesion social, educación y convivencia intercultural & OP & 6 \\
\hline \multirow{4}{*}{ U. Granada } & 4. Sociología de las migraciones. & OP & 6 \\
\hline & 5. Bases Psicológicas de la Educación Inter e Intracultural (Campus Ceuta) & OP & 4,5 \\
\hline & 6. Didáctica de la comunicación lingüística como instrumento de mediación intercultural (Campus Ceuta) & OP & 6 \\
\hline & 7. Diseño y desarrollo de programas interculturales (Campus Melilla) & OP & 6 \\
\hline \multirow{3}{*}{ U. Huelva } & 8. Pluralismo cultural y Convivencia & OP & 6 \\
\hline & 9. Educación Intercultural & OP & 6 \\
\hline & 10. Competencia comunicativa para la interculturalidad & OP & 3 \\
\hline U. Málaga & 11. Inmigración, minorías étnicas y educación intercultural & OP & 6 \\
\hline U. Pablo de Olavide & 12. La educación social ante la diversidad cultural & $O B$ & 6 \\
\hline U. Las Palmas de Gran Canaria & 13. Educación en contextos de multiculturalidad & $O B$ & 6 \\
\hline \multirow{2}{*}{ U. Castilla-La Mancha } & 14. Interculturalidad y Educación & $O B$ & 6 \\
\hline & 15. Teoría social de las migraciones y de la exclusión social & $O B$ & 6 \\
\hline U. Burgos & 16. Diversidad socio-cultural & $O B$ & 6 \\
\hline U. León & 17. Inmigrantes, minorías étnicas y educación intercultural & $O B$ & 6 \\
\hline U. Salamanca & 18. Interculturalismo, Género y Educación & $O B$ & 6 \\
\hline U. Valladolid & 19. Inmigrantes, Minorías Étnicas y Educación Intercultural & $O B$ & 6 \\
\hline U. Autónoma de Barcelona & 20. Acogida e Inclusión de Personas Inmigrantes & OP & 6 \\
\hline U. Girona & 21. La mediación comunitaria en contextos multiculturales & $O P$ & 3 \\
\hline \multirow{4}{*}{ U. Lleida } & 22. Inmigración y sociedad multicultural & OP & 6 \\
\hline & 23. Enfoque y modelos de Educación Multicultural & OP & 6 \\
\hline & 24. Proyectos de Educación Multicultural & OP & 6 \\
\hline & 25. Selección y diseño de materiales para la Educación Multicultural & OP & 6 \\
\hline \multirow[t]{2}{*}{ U. Rovira I Virgili } & 26. Diagnóstico, investigación e Intervención en contextos multiculturales & OP & 6 \\
\hline & 27. Ética en la investigación socioeducativa en contextos multiculturales & OP & 6 \\
\hline
\end{tabular}




\begin{tabular}{|c|c|c|c|}
\hline UNIVERSIDAD & ASIGNATURAS & CARÁCTER & CRÉDITOS \\
\hline \multirow{4}{*}{ U. Complutense de Madrid } & 28. Educación y mediación intercultural & OB & 6 \\
\hline & 29. Sociología de la inmigración & OP & 6 \\
\hline & 30. Programas de educación intercultural & OB & 6 \\
\hline & 31. Medios audiovisuales y educación intercultural & OP & 6 \\
\hline U. Alcalá & 32. Inmigración y diversidad cultural & OB & 6 \\
\hline UNED & 33. Educación Intercultural & OP & 6 \\
\hline U. Extremadura & 34. Educación intercultural & OP & 6 \\
\hline U. Coruña & 35. Educación multicultural & OB & 6 \\
\hline U. Santiago de Compostela & 36. Educación Social, Diversidad Cultural y Desarrollo Local & OP & 6 \\
\hline U. Vigo & 37. Educación intercultural y para la paz & OP & 6 \\
\hline U. Islas Baleares & 38. Educación Intercultural, Intervención Socioeducativa e Inmigración & OB & 6 \\
\hline U. País Vasco & 39. Educación intercultural (Campus Guipúzcoa) & OP & 6 \\
\hline
\end{tabular}

Fuente: elaboración propia a partir de datos obtenidos en páginas web.

Además, nos parece importante destacar que solo en dos de las universidades en las que se oferta el grado de Educación Social, existen menciones específicas que hacen alguna alusión a las temáticas que nos ocupan. La Universidad de Granada, con el itinerario de especialización en «Educación social, interculturalidad y globalización» y en la Universidad Rovira i Virgili con la Mención en «Intervención socioeducativa en contextos multiculturales».

A continuación, en cuanto a relevancia de la oferta educativa en el tema que nos ocupa, se encuentra el Grado de Educación Primaria. En 20 de las 39 universidades que cuentan con este grado existen asignaturas sobre la temática, con un total de 35 asignaturas ofertadas. De dichas asignaturas solo el 14\% (5 del total) son obligatorias y entre todas representan tan solo el 3,7\% del total de los créditos ofertados por dichas universidades, oscilando entre el 1,2\% de la Universidad de Navarra o de la Universidad de Las Palmas (que ofertan solo una asignatura optativa de 3 créditos en esta línea) y la amplia oferta de la Universidad del País Vasco (con 37,5 créditos especializados repartidos en 7 asignaturas) o de la Universidad de Murcia (que ofrece 6 asignaturas con un total de 36 créditos) y que incluye unas prácticas de 15 créditos que posibilitan la especialización en Educación Intercultural. Véase la Tabla II.

Tabla II. Asignaturas sobre «interculturalidad» y/o «migraciones» ofertadas para los estudios de Grado de Educación Primaria en las universidades públicas españolas (Curso 2018-19)

\begin{tabular}{|c|c|c|c|}
\hline UNIVERSIDAD & ASIGNATURAS & CARÁCTER & CRÉDITOS \\
\hline U. Jaén & 1. Descubrir y valorar el patrimonio histórico-artístico y la diversidad cultural & OP & 6 \\
\hline U. Córdoba & 2. El desarrollo de la competencia multilingüe-multicultural & OP & 6 \\
\hline U. Sevilla & 3. Análisis y Producción de Textos en Lengua Inglesa y Educación Intercultural & OP & 6 \\
\hline U. Málaga & 4. Intervención Educativa y Diversidad Sociocultural & OP & 6 \\
\hline U. Alcalá & 5. Interculturalidad y recursos tecnológicos & OP & 6 \\
\hline U. Barcelona & 6. Sociología de la Educación, cambios sociales, educativos y multiculturalidad & $O B$ & 6 \\
\hline \multirow{3}{*}{ U. Girona } & 7. Arte, calidad y multiculturalidad & OP & 3 \\
\hline & 8. Educación estética y vida cotidiana: postmoderna, multicultural, feminista, ecológica & OP & 3 \\
\hline & 9. Del patrimonio multicultural a la contemporaneidad artística: recursos & OP & 3 \\
\hline \multirow{5}{*}{ U. Rovira I Virgili } & 10. Competencia comunicativa e intercultural en lengua extraniera & OP & 6 \\
\hline & 11. Atención a la diversidad social, lingüística y cultural de la escuela & OP & 3 \\
\hline & 12. Fundamentos teóricos de la Educación Intercultural (Campus Álava) & OP & 6 \\
\hline & 13. La dimensión comunitaria de la interculturalidad (Campus Álava) & OP & 4,5 \\
\hline & 14. La interculturalidad en el ámbito escolar (Campus Álava) & OP & 4,5 \\
\hline
\end{tabular}




\begin{tabular}{|c|c|c|c|}
\hline UNIVERSIDAD & ASIGNATURAS & CARÁCTER & CRÉDITOS \\
\hline \multirow{4}{*}{ U. País Vasco } & 15. Políticas educativas para la educación intercultural (Campus Állava) & OP & 4,5 \\
\hline & 16. Recursos y estrategias didácticas para la educación intercultural (Campus Álava) & OP & 6 \\
\hline & 17. Didáctica de segundas lenguas a alumnado inmigrante (Campus Vizcaya) & OP & 6 \\
\hline & 18. Comunicación intercultural (Campus Guipúzcoa) & OP & 6 \\
\hline U. Zaragoza & 19. Educación social e intercultural & OB & 6 \\
\hline \multirow{4}{*}{ U. Coruña } & 20. Educación inclusiva y multicultural & OB & 6 \\
\hline & 21. Educación intercultural y atención a la diversidad & OP & 6 \\
\hline & 22. Educación antirracista & OP & 3 \\
\hline & 23. Aprendizaje cooperativo e interculturalidad & OP & 3 \\
\hline \multirow{3}{*}{ U. Murcia } & 24. Educación ante una realidad pluricultural & OP & 6 \\
\hline & 25. Procesos y estrategias educativas en una realidad intercultural & OP & 3 \\
\hline & 26. Prácticas escolares III Educación intercultural & OP & 15 \\
\hline U. Las Palmas de Gran Canaria & 27. Aulas y escuelas interculturales & OP & 3 \\
\hline \multirow{2}{*}{ U. Islas Baleares } & 28. Educación Intercultural & OP & 3 \\
\hline & 29. Atención a la Diversidad Social, Lingǘstica y Cultural en la Escuela & OP & 3 \\
\hline U. Navarra & 30. Educación Intercultural & OP & 3 \\
\hline U. Cantabria & 31. La escuela intercultural. Investigaciones, experiencias y materias & OP & 6 \\
\hline U. La Rioja & 32. La acción socioeducativa en contextos multiculturales & OP & 4,5 \\
\hline U. Castilla-La Mancha & 33. Tratamiento educativo de las necesidades procedentes de la diversidad cultural & OP & 6 \\
\hline U. Burgos & 34. Sociología de la Educación, Interculturalidad e Inclusión Social & OB & 6 \\
\hline U. Valladolid & 35. Cambios Sociales, Cambios Educativos e Interculturalidad & OB & 6 \\
\hline
\end{tabular}

Fuente: elaboración propia a partir de datos obtenidos en páginas web.

En tercer lugar, encontramos el Grado de Educación Infantil. De las 39 universidades que lo ofrecen, solo 19 incluyen alguna asignatura relacionada con la interculturalidad o las migraciones, con un total de 28 en todas las universidades, de las cuales, como se muestra en la Tabla III, solo 6 son de carácter obligatorio. En total, supone una media de un $3,4 \%$ de la carga lectiva total.

\section{Tabla III. Asignaturas sobre «interculturalidad» y/o «migraciones» ofertadas para los estudios de Grado de Educación Infantil en las universidades públicas españolas (Curso 2018-19)}

\begin{tabular}{|c|c|c|c|}
\hline UNIVERSIDAD & ASIGNATURAS & CARÁCTER & CRÉDITOS \\
\hline U. Almería & 1. Deporte, multiculturalidad e integración social & $\mathrm{OP}$ & 6 \\
\hline U. Jaén & 2. Descubrir y valorar el patrimonio histórico-artístico y la diversidad cultural & OP & 6 \\
\hline U. Córdoba & 3. Atención psicoeducativa a la diversidad cultural & $\mathrm{OP}$ & 6 \\
\hline \multirow{4}{*}{ U. Granada } & 4. Diversidad, interculturalidad y educación inclusiva & OP & 6 \\
\hline & 5. Currículum Intercultural (Campus Melilla) & OP & 6 \\
\hline & 6. Plurilingüismo en contextos educativos multiculturales (Campus Melilla) & $\mathrm{OP}$ & 6 \\
\hline & 7. Diseño y desarrollo de programa educativos interculturales (Campus Melilla) & $\mathrm{OP}$ & 6 \\
\hline U. Huelva & 8. Educación Intercultural & $O P$ & 6 \\
\hline U. Alcalá & 9. Interculturalidad y recursos tecnológicos & $\mathrm{OP}$ & 6 \\
\hline U. Complutense de Madrid & 10. La dimensión intercultural en el currículum & OP & 6 \\
\hline U. Barcelona & 11. Sociología de la Educación, cambios sociales, educativos y multiculturalidad & OB & 6 \\
\hline \multirow{2}{*}{ U. Girona } & 12. Educación estética y vida cotidiana: postmoderna, multicultural, feminista, ecológica. & $\mathrm{OP}$ & 3 \\
\hline & 13. Del patrimonio multicultural a la contemporaneidad artística: recursos & $\mathrm{OP}$ & 3 \\
\hline U. Lleida & 14. Juegos, valores y diversidad culturall & OP & 6 \\
\hline \multirow{3}{*}{ U. Rovira I Virgili } & 15. Intervenció Psicoeducativa en la Diversitat Social, Ètnica i Cultural & OP & 6 \\
\hline & 16. La dimensión comunitaria de la interculturalidad (Campus Álava) & $\mathrm{OP}$ & 4,5 \\
\hline & 17. La interculturalidad en el ámbito escolar (Campus Álava) & $O P$ & 4,5 \\
\hline
\end{tabular}




\begin{tabular}{|c|c|c|c|}
\hline UNIVERSIDAD & ASIGNATURAS & CARÁCTER & CRÉDITOS \\
\hline \multirow{3}{*}{ U. País Vasco } & 18. Políticas educativas para la educación intercultural (Campus Álava) & OP & 4,5 \\
\hline & 19. Recursos y estrategias didácticas para la educación intercultural (Campus Álava) & OP & 6 \\
\hline & 20. Didáctica de segundas lenguas a alumnado inmigrante (Campus Vizcaya) & OP & 6 \\
\hline U. Zaragoza & 21. Educación social e intercultural & OB & 6 \\
\hline U. A Coruña & 22. Educación inclusiva y multicultural & OB & 6 \\
\hline U. Navarra & 23. Educación Intercultural & OP & 3 \\
\hline U. Cantabria & 24. La escuela intercultural. Investigaciones, experiencias y materiales & OP & 6 \\
\hline U. Salamanca & 25. Interculturalismo, género y educación & OB & 6 \\
\hline U. Burgos & 26. Educación intercultural, para la paz y la igualdad & OB & 9 \\
\hline U. Valladolid & 27. Educación Intercultural & OB & 6 \\
\hline U. León & 28. Educación intercultural & OP & 4 \\
\hline
\end{tabular}

Fuente: elaboración propia a partir de datos obtenidos en páginas web.

Y por último se encuentra el Grado de Pedagogía, impartido en 19 universidades de las cuales, solo 12 incluyen materias vinculadas a las temáticas que estamos trabajando. De las 15 asignaturas que hemos recopilado, tan solo 4 son de carácter obligatorio, y es de destacar que ninguna universidad incluye más de 12 créditos en estos asuntos (al menos, no directamente), y tan solo suponen el 2,8\% de los créditos totales ofertados por las distintas universidades. Véase la Tabla IV.

\section{Tabla IV. Asignaturas sobre «interculturalidad» y/o «migraciones» ofertadas para los estudios de Grado de Pedagogía en las universidades públicas españolas (Curso 2018-19)}

\begin{tabular}{|c|c|c|c|}
\hline UNIVERSIDAD & ASIGNATURAS & CARÁCTER & CRÉDITOS \\
\hline U. Granada & 1. Migraciones y educación & OP & 6 \\
\hline \multirow{3}{*}{ U. Girona } & 2. Cultura, inmigración y educación & OP & 3 \\
\hline & 3. Pedagogía e interculturalidad & OP & 3 \\
\hline & 4. Nuestros contextos de diversidad cultural & OP & 3 \\
\hline U. Rovira I Virgili & 5. Intervenció Psicoeducativa en la Diversitat cultural en el marc d’una Educació Inclusiva & OP & 6 \\
\hline U. Valencia & 6. Pedagogía Intercultural & $O B$ & 6 \\
\hline U. Santiago de Compostela & 7. Pedagogía Intercultural & OB & 6 \\
\hline U. Murcia & 8. Educación Intercultural & OP & 3 \\
\hline U. Navarra & 9. Educación Intercultural & $O P$ & 3 \\
\hline U. Islas Baleares & 10. Educación intercultural, intervención socioeducativa e Inmigración & OP & 6 \\
\hline UNED & 11. Educación Intercultural & OP & 6 \\
\hline U. Salamanca & 12. Multiculturalismo, ciudadanía y educación & $O B$ & 6 \\
\hline U. Oviedo & 13. Pedagogía ambiental e intercultural & $O P$ & 6 \\
\hline \multirow{2}{*}{ U. Burgos } & 14. Multiculturalidad, Interculturalidad e Inclusión Social & $O B$ & 6 \\
\hline & 15. Comunicación Intercultural y Diseño de Recursos Socioeducativos & $\mathrm{OP}$ & 6 \\
\hline
\end{tabular}

Fuente: elaboración propia a partir de datos obtenidos en páginas web.

En total se han contabilizado 117 asignaturas. En ciertas universidades se repiten algunas (sobre todo en la oferta de optativas), con lo que este número hace referencia a las materias observadas en el total de los grados indicados, independientemente de que sea la misma asignatura para infantil y primaria, o en distintos campus, por ejemplo. La decisión de cuantificarlas de esta manera tiene que ver con el hecho de que, aunque se trate en ocasiones de la misma materia, el alumnado que la cursa es diferente.

En cuanto a las características del conjunto de las asignaturas observadas, en primer lugar, hay que señalar que la gran mayoría son materias semestrales de 6 créditos (85 de ellas, con la excepción de unas 
prácticas de 15 créditos que duran también un semestre) - en la línea de la mayoría de las materias existentes en la actualidad en los estudios de grado en España-; hay algunas semestrales de 4 o 4.5 créditos (9 asignaturas); de 3 créditos (21 asignaturas); y tan solo una anual (una de 9 créditos). El 77\% de las asignaturas registradas son de carácter optativo (90 de ellas), por lo que tan solo un 23\% (27) son asignaturas obligatorias (bien básicas, bien troncales u obligatorias estrictamente hablando). Esta característica hace que además la mayoría de ellas se oferten en los últimos cursos de los grados ( $3^{\circ}$ y $4^{\circ}$ principalmente), dado que la optatividad no suele situarse en los primeros años de carrera. Los departamentos encargados de impartirlas son principalmente los de Pedagogía, Sociología, Psicología, Antropología y Didáctica. No obstante, al no ser esta una información siempre ofrecida en las webs consultadas resulta complicado, en estos momentos de aproximación al tema, ofrecer información cuantitativa al respecto.

\section{Discusión: ¿̇es importante la formación inicial en migraciones e interculturalidad para futuros/as profesionales de lo social?}

Después de la revisión realizada puede afirmarse que actualmente en la universidad pública española existe una oferta amplia (aunque ciertamente sesgada por la orientación optativa y el bajo número de créditos asignados a las mismas) de materias que contemplan la formación para futuras/os profesionales de la educación en materias relacionadas con la «interculturalidad» y las «migraciones» de manera explícita. El elevado porcentaje de universidades que incluyen al menos una asignatura de este tipo en alguno de los 4 grados analizados, un $85 \%$ de la muestra, avala suficientemente esta afirmación.

A este porcentaje se sumaría aquella formación no contemplada al realizar la muestra y que incluye referencias a educación intercultural y/o migraciones en el detalle de los contenidos de asignaturas denominadas «Educación Inclusiva», «Antropología de la Educación» o «Atención a la diversidad», entre otras. El no analizar asignaturas que no contuviesen en el título alguno de los términos de búsqueda establecidos, ha limitado la muestra, pero también nos ha servido para mostrar la poca visibilidad explicita de estas temáticas en formación obligatoria. Al situar la mayor oferta en la optatividad, pareciera que se trata de una «especialidad» y no de una realidad a la que tendrán que hacer frente todas las personas que se dediquen a la educación formal y no formal y que hoy estudian grados relacionados.

Revisando los datos y comparándolos con años anteriores, si bien la oferta se ha ampliado levemente ( 2 asignaturas nuevas en 5 años), nos ha resultado sorprendente que la Universidad de Almería ${ }^{3}$ haya eliminado de su plan de estudios para los grados de educación primaria e infantil, varias asignaturas cuyo título relacionaba directamente la intervención educativa, las migraciones y la interculturalidad, más siendo un contexto especialmente significativo en el trabajo con poblaciones migrantes en el sur de España. Aquí las estrategias de las Universidades objeto de este trabajo, parecen no tener una correlación directa con las políticas públicas o los contextos concretos, pues otro ejemplo de ello lo tenemos en la Universidad de Granada, que para el campus de Melilla despliega una oferta amplia orientada al trabajo educativo en contextos multiculturales y en cambio, para el campus de Ceuta dicha oferta en los distintos grados se reduce a la mitad.

Como veíamos, el grado que otorga mayor importancia a la formación en «interculturalidad» y «migraciones» es el Grado de Educación Social. Como sabemos, el ámbito de trabajo de los/as educadores/

\footnotetext{
3 Nos parece de especial relevancia, dado que Almería fue el primer lugar en España en adoptar medidas dirigidas especialmente a la población inmigrante desde la Consejería de Educación de la Junta de Andalucía, nos referimos a las Aulas Temporales de Adaptación Lingüística (ATAL) en los años 90 del pasado siglo. Además de otras iniciativas que han ido adoptándose y en las que ha sido pionera.
} 
as sociales no suele ser la educación formal. Esto es importante en tanto que podemos concluir que, a pesar de que la formación en dichas materias en los estudios de grado actuales de la universidad pública española es significativa, se está entendiendo que dichas temáticas serán útiles sobre todo para profesionales de la educación que trabajen «fuera de la escuela». Es decir, la capacidad de modificación del sistema educativo a partir de la inclusión de nuevas perspectivas y contenidos curriculares adaptados a la actual situación de multiculturalidad, se está viendo en cierto modo reducida: no son los/as futuros/as maestros/as quienes en mayor medida están recibiendo actualmente formación para ello. Pero sí son las/ os futuras/os maestras/os quienes tendrán que gestionar aulas diversas social y culturalmente. Y especialmente relevante es la presencia más reducida de dichas materias en los estudios de Grado de Pedagogía, porque los/as pedagogos/as serían el perfil profesional especializado y capacitado para el diseño de planes formativos y enfoques curriculares que ayudasen en el proceso de interculturalización del sistema educativo español. No somos capaces de adelantar situaciones venideras, pero sí creemos que esta falta de competencias interculturales a nivel curricular puede hacer mella en la formación en Pedagogía que las/os estudiantes reciben y que no facilita la comprensión y el trabajo sobre la realidad multicultural que existe hoy en nuestras escuelas.

Por otro lado, nos gustaría destacar que existen algunas universidades, algunos grados, que tienen una oferta formativa sobre «interculturalidad» $\mathrm{y} / \mathrm{o}$ «migraciones» interesante, amplia e innovadora, en tanto que consiguen recoger e interrelacionar contenidos y competencias - muchas de ellas sugeridas de manera reiterada en las diferentes investigaciones sobre educación y formación intercultural- que tienen que ver, entre otras cuestiones, con: la didáctica, la intervención socio-psicológica, el diseño de currículos o la sensibilización y la formación en valores. Es decir, dan cobertura al estudio y análisis de la diversidad cultural desde distintos enfoques disciplinares, lo cual es muy enriquecedor de cara a conseguir una formación adecuada y lo más holista posible para los y las futuros/as profesionales de la educación.

Por último y para finalizar volvemos a destacar, a modo de reflexión final y por su importancia la necesidad de que este tipo de formación sea obligatoria, explícita y permanente; algo que contrasta con la realidad de que la mayoría de las asignaturas de la muestra sea de carácter optativo. Con ello sigue quedando en evidencia cómo es el/la futuro/a profesional quien decide, en función de su sensibilidad o motivación, si formarse o no para conseguir competencias que le permitan trabajar mejor en contextos de diversidad cultural. Ello desde luego contradice la idea de una Educación Intercultural como un enfoque que ha de ser transversal. En otras palabras, si cursar estas materias sigue siendo opcional -en definitivasólo estarán preparadas/os para trabajar desde esta perspectiva aquellos/as estudiantes ya sensibilizados o motivados, y no la totalidad de las/os futuras/os maestros/as, pedagogos/as y educadores/as sociales que desempeñarán sus funciones profesionales en contextos educativos diversos culturalmente. La sociedad española es multicultural de hecho, siempre lo ha sido, pero parece que hemos tomado consciencia de ello en las últimas décadas con la presencia de poblaciones relacionadas con la migración. Lejos de ser anecdótico, de la preparación que tengan las/os futuras/os profesionales de la educación (formal y no formal) va a depender en cierta manera la construcción de un modelo social intercultural, de reconocimiento y puesta en valor de toda la diversidad o la consolidación de modelos asimilacionistas, fagocitadores de diversidad o incluso abiertamente segregadores (tendencias que hoy, más que nunca, con el auge de los fascismos, nos vemos obligadas a enfrentar). La universidad pública española, desde su clara labor y responsabilidad social, debe pronunciarse y valorar la integración de contenidos en la formación universitaria que apuesten por una sociedad equitativa, justa, plural, diversa y que los desarrollen apor- 
tando herramientas a futuras/os profesionales de la educación para fomentar una convivencia realmente intercultural.

\section{Referencias bibliográficas}

Carrasco Pons, Silvia (2011): “Interculturalidad e inclusión: principios para evaluar la acogida al alumnado de origen extranjero” en F. Javier García Castaño y Silvia Carrasco Pons (Eds.). Población inmigrante y escuela: conocimientos y saberes de investigación. Madrid: Ministerio de Educación.

Consejería de Empleo, Dirección General de Coordinación de Políticas Migratorias (2009). Proyecto Forinter2: Formación en Interculturalidad y Migraciones. Programa Operativo del Fondo Social Europeo para Andalucía 2007-2013. Junta de Andalucía.

Consejería de Gobernación, Dirección General de Coordinación de Políticas Migratorias. (2006). Proyecto Forinter: Formación en Interculturalidad. Programa Operativo Integrado de Andalucía 2000-2006. Sevilla: Junta de Andalucía.

Dietz, Gunther (2012). Multiculturalismo, Interculturalidady diversidad en educación: una aproximación antropológica. México: Fondo de Cultura Económica.

De la Torre, Eva (2018). Universidades públicas y privadas: evolución de la productividad e impacto de la crisis. Observatorio Social de la Caixa (en línea). https://observatoriosociallacaixa.org/es/-/ universidades-publicas-y-privadas, consultado el 2 de abril de 2019.

Essomba, Miguel Ángel (2006). Liderar escuelas interculturales e inclusivas. Equipos directivos y profesorado ante la diversidad cultural y la inmigración. Barcelona: Graó

Franzé Mudanó, Adela (2003): “Imágenes y prácticas de los usos de la interculturalidad en el campo educativo” en Asunción Barañano y Jose Luis García (Coords.). Culturas en contacto: encuentros y desencuentros. Madrid: Ministerio de Educación, Cultura y Deporte.

García Castaño, F. Javier y Pulido Moyano, Rafael (2008): "El desarrollo de la antropología de la educación en España: razones que explican la casi monográfica mirada a las llamadas minorías étnicas” en María Isabel Jociles Rubio y Adela Franzé Mudanó (Eds.). ¿Es la escuela el problema?: perspectivas socioantropológicas de etnografía y educación. Madrid: Trotta.

García Castaño, F. Javier, Fernández Echeverría, José, Rubio Gómez, María y Soto Páez, Lourdes (2011): "Inmigración extranjera y educación en España: algunas reflexiones sobre el 'alumnado de nueva incorporación’” ” en F. Javier García Castaño y Silvia Carrasco Pons (Eds.). Población inmigrante y escuela: conocimientos y saberes de investigación. Madrid: Ministerio de Educación.

García Castaño, F. Javier, Granados Martínez, Antolín, y García-Cano Torrico, María (1999): “De la educación multicultural e intercultural a la lengua y cultura de origen: reflexiones sobre el caso español” en Adela Franzé (Coord.). Lengua y cultura de origen. Madrid: Ediciones del Oriente y del Mediterráneo.

García Castaño, F. Javier, Granados Martínez, Antolín y Pulido Moyano, Rafael (1999): “Reflexiones en diversos ámbitos de construcción de la diferencia” en F. Javier García Castaño y Antolín Granados Martínez (Eds.). Lecturas para educación intercultural. Madrid: Trotta.

García Castaño, F. Javier, Rubio Gómez, María y Fernández Echeverría, José (2018): “Las trampas de la diversidad. Sobre la producción de diferencias en la escuela”. Gažeta de Antropología, 31, 61-86. 
García Castaño, F. Javier, Olmos Alcaraz, Antonia, Contini, Pierangela, y Rubio Gómez, María (2012): "Sobre multiculturalismo, críticas y superaciones conceptuales en la gestión de la diversidad cultural" en Estrella Gualda (Coord.). Inmigración, ciudadanía y gestión de la diversidad. Sevilla: Universidad Internacional De Andalucía.

Jordán, Jose Antonio (1992): "Educación multicultural, conceptos y problemáticas" en Paciano Fermoso (Ed.). Educación Intercultural: la Europa sin fronteras. Barcelona: Narcea

Leiva, Juan José (2014): “La interculturalidad en el contexto universitario a través de las voces de estudiantes inmigrantes”. Revista Electrónica Interuniversitaria de Formación del Profesorado, 17 (2), 155-166.

Leiva, Juan José (2012): “La formación en educación intercultural del profesorado y la comunidad educative”. Revista Electrónica de Investigación y Docencia (REID), 10, 8-31.

Martinez Chicón, Raquel (2011): "Aprendiendo a gestionar la diversidad en las adminsitraciones públicas” en F.J. García Castaño y N. Kressova (Coord.). Actas del I Congreso Internacional sobre Migraciones en Andalucía. Granada: Universidad de Granada.

Martínez Chicón, Raquel y Olmos Alcaraz, Antonia (2014): “Competencias interculturales en el Grado de Trabajo Social: una 'evaluación alternativa"” en E. Pastor (coord.). El trabajo social ante el reto de la crisisy la educación superior. Murcia: Universitas.

Olmos Alcaraz, Antonia (2010): "Construcción discursiva del alumno inmigrante extranjero en Andalucía: el otro en nuestras escuelas”, Revista de Educación, 469-493.

Olmos Alcaraz, Antonia y Martínez Chicón, Raquel (2015): “¿Gestionar diversidades o diferencias? Interculturalismo, educación y sanidad en España y EE.UU.”. Aposta Revista de Ciencias Sociales, 66, 1-20.

Peñalva, Alicia y Soriano, Encarnación (2010): “Objetivos y contenidos sobre interculturalidad en la formación inicial de educadores y educadoras”. Estudios sobre Educación, 18, 37-57.

Rodrigo Alsina, Miguel (1997): "Elementos para una comunicación intercultural”. Revista CIDOB d'afers internacionals, 36, 11-21.

Rodrigo Alsina, Miguel (1999). Comunicación intercultural. Barcelona: Anthropos.

Rodríguez Izquierdo, Rosa María (2009): “La investigación sobre la educación intercultural en España”. Archivos Analiticos de Politicas Educativas, 17 (4), 1-26. http://dx.doi.org/10.14507/epaa.26.3461.

Vázquez Aguado, Octavio (2009): “La competencia intercultural y las habilidades de intervención en mediación social intercultural” en Consejería de Empleo (Ed.). Salud, Materiales Didácticos. Proyecto Forinter. Sevilla: Junta de Andalucía.

\section{Notas biográficas}

María Rubio Gómez es Doctora en Antropología Social por la Universidad de Granada (2013), Licenciada en Antropología Social y Máster en Estudios Migratorio, Desarrollo e Intervención Social. Investigadora del Instituto de Migraciones y del Grupo de Investigación Laboratorio de Estudios Interculturales (SEJ-123). Su trayectoria profesional está vinculada a la intervención social y la Educación para el Desarrollo en el tercer sector y a la investigación sobre alteridad/otredad en contextos educativos. Sus líneas de investigación son, construcción de alteridad y gestión de la diversidad vinculada con el fenómeno 
migratorio en la escuela, educación intercultural, racismo y exclusión/inclusión social. Actualmente es profesora en el Departamento de Antropología Social de la Universidad de Granada.

Raquel Martinez Chicón es Doctora en Antropología Social y Cultural por la Universidad de Granada, Licenciada en Antropología Social y Cultural por la Universidad de Sevilla y Diplomada en Trabajo Social por la Universidad de Granada. Profesora Contratada Doctora del Departamento de Antropología Social de la Universidad de Granada e Investigadora del Instituto de Migraciones de la Universidad de Granada (España). Investigadora Principal del proyecto I+D+i: «Menores migrantes en el arco mediterráneo: movilidad, sistemas de acogida e integración» (DER2017-89623-R). Ministerio de Economía, Industria y Competitividad. Convocatoria 2017. Líneas de investigación: Migraciones, Interculturalidad y Gestión de la Diversidad Cultural.

Antonia Olmos Alcaraz es Doctora en Antropología Social por la Universidad de Granada (2009), Licenciada en Sociología, Máster en Inserción Socio-laboral, Minorías y Territorio. Investigadora en el Instituto de Migraciones de la Universidad de Granada, y parte del equipo de investigación del «Laboratorio de Estudios Interculturales» (SEJ123). Sus líneas de investigación son migraciones, alteridad/identidad, educación intercultural y discurso político/mediático sobre la inmigración. Actualmente es profesora en el Departamento de Antropología Social de la Universidad de Granada, desde donde imparte las materias de Antropología de la Educación y Migraciones y Educación. 